Acta Crystallographica Section F

Structural Biology

and Crystallization

Communications

ISSN 1744-3091

Constantina Bakolitsa, ${ }^{\text {a,b }}$ Alex Bateman, ${ }^{\mathrm{c}}$ Kevin K. Jin, ${ }^{\mathrm{a}, \mathrm{d}}$ Daniel McMullan, ${ }^{a, e}$ S. Sri Krishna,, a,b,f Mitchell D. Miller, ${ }^{\mathrm{a}, \mathrm{d}}$ Polat Abdubek, ${ }^{\mathrm{a}, \mathrm{e}}$ Claire Acosta, ${ }^{a, e}$ Tamara Astakhova, ${ }^{a, f}$ Herbert L. Axelrod, ${ }^{a, d}$ Prasad Burra, ${ }^{a, b}$ Dennis Carlton, ${ }^{a, g}$ Hsiu-Ju Chiu, ${ }^{\text {a,d }}$ Thomas Clayton, ${ }^{\mathrm{a}, \mathrm{g}}$ Debanu Das, ${ }^{\mathrm{a}, \mathrm{d}}$ Marc C. Deller, ${ }^{a, g}$ Lian Duan, ${ }^{a, f}$ Ylva Elias, ${ }^{\text {a,g }}$ Julie Feuerhelm, ${ }^{\text {a,e }}$ Joanna C. Grant, ${ }^{\text {a,e }}$ Anna Grzechnik, a,g Slawomir K. Grzechnik, ${ }^{\text {, f }}$ Gye Won Han, ${ }^{a, g}$ Lukasz Jaroszewski, ${ }^{a, b, f}$ Heath E. Klock, ${ }^{a, e}$ Mark W. Knuth, Piotr Kozbial, a,b Abhinav Kumar, David Marciano, ${ }^{\mathrm{a}, \mathrm{g}}$ Andrew T. Morse, ${ }^{a, f}$ Kevin D. Murphy, ${ }^{\text {a,g }}$ Edward Nigoghossian, ${ }^{\mathrm{a}, \mathrm{e}}$ Linda Okach, ${ }^{\mathrm{a}, \mathrm{e}}$ Silvya Oommachen, ${ }^{a, d}$ Jessica Paulsen, ${ }^{a, e}$ Ron Reyes, ${ }^{a, d}$ Christopher L. Rife, ${ }^{a, d}$ Natasha Sefcovic, ${ }^{a, b}$ Henry Tien, ${ }^{a, f}$ Christine B. Trame, ${ }^{\mathrm{a}, \mathrm{d}}$ Christina V. Trout, ${ }^{a, g}$ Henry van den Bedem, ${ }^{a, d}$ Dana Weekes, ${ }^{a, b}$ Aprilfawn White, ${ }^{a, e}$ Qingping Xu, ${ }^{\text {a,d }}$ Keith O. Hodgson, ${ }^{\text {a,h }}$ John Wooley, ${ }^{\text {,af }}$ Marc-André Elsliger, ${ }^{a, g}$ Ashley M. Deacon, ${ }^{\text {a,d }}$ Adam Godzik, ${ }^{a, b, f}$ Scott Lesley ${ }^{a, e, g}$ and Ian A. Wilson ${ }^{a, g_{*}}$

aJoint Center for Structural Genomics, http:// www.jcsg.org, USA, ${ }^{\mathbf{b}}$ Program on Bioinformatics and Systems Biology, Burnham Institute for Medical Research, La Jolla, CA, USA, ${ }^{c}$ Wellcome Trust Sanger Institute, Wellcome Trust Genome Campus, Hinxton CB10 1SA, England, ${ }^{\mathbf{d}}$ Stanford Synchrotron Radiation Lightsource, SLAC National Accelerator Laboratory, Menlo Park, CA, USA, ${ }^{\mathbf{e}}$ Protein Sciences Department, Genomics Institute of the Novartis Research Foundation, San Diego, CA, USA, 'Center for Research in Biological Systems, University of California, San Diego, La Jolla, CA, USA ${ }^{\mathrm{g}}$ Department of Molecular Biology, The Scripps Research Institute, La Jolla, CA, USA, and hPhoton Science, SLAC National Accelerator Laboratory, Menlo Park, CA, USA

Correspondence e-mail: wilson@scripps.edu

Received 10 June 2009

Accepted 29 June 2009

PDB Reference: Jann_2411 from Jannaschia sp. strain CCS1, 3h0n, r3h0nsf.

\section{The structure of Jann_2411 (DUF1470) from Jannaschia sp. at $1.45 \AA$ resolution reveals a new fold (the ABATE domain) and suggests its possible role as a transcription regulator}

The crystal structure of Jann_2411 from Jannaschia sp. strain CCS1, a member of the Pfam PF07336 family classified as a domain of unknown function (DUF1470), was solved to a resolution of $1.45 \AA$ by multiple-wavelength anomalous dispersion (MAD). This protein is the first structural representative of the DUF1470 Pfam family. Structural analysis revealed a two-domain organization, with the N-terminal domain presenting a new fold called the ABATE domain that may bind an as yet unknown ligand. The C-terminal domain forms a treble-clef zinc finger that is likely to be involved in DNA binding. Analysis of the Jann_2411 protein and the broader ABATE-domain family suggests a role as stress-induced transcriptional regulators.

\section{Introduction}

The complete genome sequences of hundreds of organisms are now known, each of which contains thousands of genes that have evolved to create the bewildering diversity of life. To understand this complexity at the molecular level requires the investigation of the function and structure of a vast number of proteins. A major goal of the Protein Structure Initiative (PSI; http://www.nigms.nih.gov/Initiatives/ $\mathrm{PSI} /$ ) is to expand our knowledge of the protein universe by solving the structures of representative members of large as yet uncharacterized protein families. The Pfam database (Finn et al., 2008) contains over 2000 such families, termed domains of unknown function (DUFs), and understanding their structure will help to elucidate their function. Thus, to extend the structural coverage of proteins with uncharacterized biological function, we targeted the Pfam protein family DUF1470 and have determined the structure of the Jann_2411 gene product from Jannaschia sp. strain CCS1, an ecologically relevant marine proteobacterium found in coastal and open surface waters.

Jann_2411 has a molecular weight of $20.7 \mathrm{kDa}$ (residues 1-187) and a calculated isoelectric point of 6.6 and its crystal structure was determined using the semiautomated high-throughput pipeline of the Joint Center for Structural Genomics (JCSG; http://www.jcsg.org; Lesley et al., 2002) as part of the NIGMS Protein Structure Initiative. Structural analysis revealed a two-domain organization, with the Nterminal domain consisting of a new fold that we call the ABATE (for Alpha-Beta-hairpin-Alpha TandEm) domain and the C-terminal domain forming a treble-clef zinc finger that we have termed the CGNR zinc-finger domain after a characteristic sequence motif that is conserved in this family. Jan_2411 forms a dimer, with both monomers implicated in the formation of a putative DNA binding site, and analysis of its genomic context suggests a role for the ABATE-domain family in stress-induced transcriptional regulation.

\section{Materials and methods}

\subsection{Protein production and crystallization}

The gene encoding Jann_2411 (GenBank YP_510353.1, gi:89054902; Swiss-Prot Q28PN4) was amplified by polymerase chain reaction (PCR) from genomic DNA using PfuTurbo DNA polymerase (Stratagene) and primers (forward primer, 5'-ctgtacttccagggcATG- 
AATTTAGACAGTTATGAAAGAACCG-3'; reverse primer, 5'-aattaagtcgcgttaTGTTGCACGACGCTCGCGAAACGCGGCG-3'; the target sequence is in upper case) corresponding to the predicted $5^{\prime}$ and $3^{\prime}$ ends. The PCR product was cloned into plasmid pSpeedET, which encodes an expression and purification tag followed by a tobacco etch virus (TEV) protease cleavage site (MGSDKIHHHHHHENLYFQ/G) at the amino-terminus of the full-length protein. The cloning junctions were confirmed by DNA sequencing. Protein expression was performed in a selenomethionine-containing medium, with suppression of normal methionine synthesis, using the Escherichia coli strain GeneHogs (Invitrogen). At the end of fermentation, lysozyme was added to the culture to a final concentration of $250 \mu \mathrm{g} \mathrm{ml}^{-1}$ and the cells were harvested. After one freeze-thaw cycle, the cells were homogenized in lysis buffer $[50 \mathrm{~m} M$ HEPES $\mathrm{pH}$ 8.0, $50 \mathrm{~m} M \mathrm{NaCl}, 10 \mathrm{~m} M$ imidazole, $1 \mathrm{~m} M$ Tris(2-carboxyethyl)phosphine hydrochloride (TCEP)] and passed through a Microfluidizer (Microfluidics). The lysate was clarified by centrifugation at $32500 \mathrm{~g}$ for $30 \mathrm{~min}$ and loaded onto nickel-chelating resin (GE Healthcare) pre-equilibrated with lysis buffer. The resin was washed with wash buffer [50 $\mathrm{m} M$ HEPES pH $8.0,300 \mathrm{~m} M \mathrm{NaCl}$, $40 \mathrm{~m} M$ imidazole, $10 \%(v / v)$ glycerol, $1 \mathrm{~m} M$ TCEP] and the protein was eluted with elution buffer [20 $\mathrm{m} M$ HEPES $\mathrm{pH} 8.0,300 \mathrm{~m} M$ imidazole, $10 \%(v / v)$ glycerol, $1 \mathrm{~m} M$ TCEP]. The eluate was bufferexchanged with HEPES crystallization buffer (20 mM HEPES pH 8.0, $200 \mathrm{~m} M \mathrm{NaCl}, 40 \mathrm{~m} M$ imidazole, $1 \mathrm{~m} M$ TCEP) using a PD-10 column (GE Healthcare) and treated with $1 \mathrm{mg}$ TEV protease per $15 \mathrm{mg}$ eluted protein. The digested eluate was passed over nickelchelating resin (GE Healthcare) pre-equilibrated with HEPES crystallization buffer and the resin was washed with the same buffer. The flowthrough and wash fractions were combined and concentrated to $12.5 \mathrm{mg} \mathrm{ml}^{-1}$ by centrifugal ultrafiltration (Millipore) for crystallization assays. Jann_2411 was crystallized using the nanodroplet vapor-diffusion method (Santarsiero et al., 2002) with standard JCSG crystallization protocols (Lesley et al., 2002). Sitting drops composed of $200 \mathrm{nl}$ protein solution mixed with $200 \mathrm{nl}$ crystallization solution were equilibrated against a $50 \mu \mathrm{l}$ reservoir at $277 \mathrm{~K}$ for $15 \mathrm{~d}$ prior to harvest. Initial screening for diffraction was carried out using the Stanford Automated Mounting system (SAM; http:// smb.slac.stanford.edu/facilities/hardware/SAM/UserInfo; Cohen et al., 2002) at the Stanford Synchrotron Radiation Lightsource (SSRL, Menlo Park, California, USA). The crystallization reagent consisted of $1.4 M$ sodium acetate and $0.1 M$ sodium cacodylate $\mathrm{pH}$ 6.5. A rodshaped crystal of approximate dimensions $150 \times 50 \times 50 \mu \mathrm{m}$ was harvested for data collection. Glycerol was added to the crystal as a cryoprotectant to a final concentration of $20 \%(v / v)$. The diffraction data were indexed in monoclinic space group C2 (Table 1). The oligomeric state of Jann_2411 in solution was determined using a $1 \times 30 \mathrm{~cm}$ Superdex 200 size-exclusion column (GE Healthcare) coupled with miniDAWN static light scattering and Optilab differential refractive index detectors (SEC/SLS; Wyatt Technology). The mobile phase consisted of $20 \mathrm{~m} M$ Tris $\mathrm{pH} 8.0,150 \mathrm{~m} M \mathrm{NaCl}$, and $0.02 \%(w / v)$ sodium azide. The molecular weight was calculated using ASTRA 5.1.5 software (Wyatt Technology).

\subsection{Data collection, structure solution and refinement}

Multiple-wavelength anomalous diffraction (MAD) data were collected at SSRL on beamline BL11-1 at wavelengths corresponding to the remote $\left(\lambda_{1}\right)$, inflection $\left(\lambda_{2}\right)$ and peak $\left(\lambda_{3}\right)$ of a selenium MAD experiment. The data sets were collected at $100 \mathrm{~K}$ with a MAR Mosaic $325 \mathrm{~mm}$ CCD detector (Rayonix, Evanston, Illinois, USA) using the Blu-Ice data-collection environment (McPhillips et al.,
Table 1

Summary of crystal parameters, data-collection and refinement statistics for Jann_2411 (PDB code 3h0n).

Values in parentheses are for the highest resolution shell.

\begin{tabular}{|c|c|c|c|}
\hline & $\lambda_{1}$ MADSe & $\lambda_{2}$ MADSe & $\lambda_{3}$ MADSe \\
\hline \multicolumn{4}{|l|}{ Data collection } \\
\hline Space group & $C 2$ & & \\
\hline Unit-cell parameters $\left(\AA{ }^{\circ},{ }^{\circ}\right)$ & \multicolumn{3}{|c|}{$a=77.75, b=59.67, c=57.82, \beta=128.8$} \\
\hline Wavelength $(\AA)$ & 0.9184 & 0.9792 & 0.9788 \\
\hline Resolution range $(\AA ̊)$ & $\begin{array}{l}25.8-1.45 \\
\quad(1.49-1.45)\end{array}$ & $\begin{array}{l}25.8-1.45 \\
\quad(1.49-1.45)\end{array}$ & $\begin{array}{l}25.8-1.45 \\
\quad(1.49-1.45)\end{array}$ \\
\hline No. of observations & 109293 & 108225 & 108208 \\
\hline No. of unique reflections & 36254 & 36192 & 36235 \\
\hline Completeness (\%) & $99.2(98.3)$ & $99.1(96.3)$ & $99.1(96.8)$ \\
\hline Mean $I / \sigma(I)$ & $19.0(4.1)$ & $17.7(3.6)$ & $17.9(3.4)$ \\
\hline$R_{\text {merge }}$ on $I \dagger(\%)$ & $4.1(29.1)$ & $4.4(30.8)$ & $4.7(34.0)$ \\
\hline \multicolumn{4}{|l|}{ Model and refinement statistics } \\
\hline Resolution range $(\AA)$ & $25.0-1.45$ & & \\
\hline No. of reflections (total) & 36254 & & \\
\hline No. of reflections (test) & 1810 & & \\
\hline Completeness (\%) & 99.1 & & \\
\hline Data set used in refinement & $\lambda_{1}$ MADSe & & \\
\hline Cutoff criterion & $|F|>0$ & & \\
\hline$R_{\text {cryst }}$ 末 & 0.140 & & \\
\hline$R_{\text {free }} \S$ & 0.157 & & \\
\hline \multicolumn{4}{|l|}{ Stereochemical parameters } \\
\hline \multicolumn{4}{|l|}{ Restraints (r.m.s. observed) } \\
\hline Bond angles $\left({ }^{\circ}\right)$ & 1.44 & & \\
\hline Bond lengths $(\AA)$ & 0.015 & & \\
\hline Average isotropic $B$ value $\left(\AA^{2}\right)$ & 16.59 & & \\
\hline ESU $\dagger \dagger$ based on $R_{\text {free }}(\AA)$ & 0.053 & & \\
\hline Protein residues/atoms & 184/1499 & & \\
\hline Waters/other molecules & $240 / 9$ & & \\
\hline
\end{tabular}

$\dagger R_{\text {merge }}=\sum_{h k l} \sum_{i}\left|I_{i}(h k l)-\langle I(h k l)\rangle\right| / \sum_{h k l} \sum_{i} I_{i}(h k l) . \quad \ddagger R_{\text {cryst }}=\sum_{h k l}|| F_{\text {obs }} \mid-$ $\left|F_{\text {calc }}\right| / \sum_{h k l}\left|F_{\text {obs }}\right|$, where $F_{\text {calc }}$ and $F_{\text {obs }}$ are the calculated and observed structure-factor amplitudes, respectively. $\& R_{\text {free }}$ is the same as $R_{\text {cryst }}$ but for $5.0 \%$ of the total reflections that were chosen at random and omitted from refinement. T This value represents the total $B$ that includes TLS and residual $B$ components. $\dagger \dagger$ Estimated overall coordinate error (Collaborative Computational Project, Number 4, 1994; Tickle et al., 1998).

2002). The MAD data were integrated and reduced using $X D S$ and scaled with XSCALE (Kabsch, 1993). Initial substructure solution was performed with SHELX (Sheldrick, 2008) and the phases were refined with SOLVE (Terwilliger \& Berendzen, 1999), with a mean figure of merit of $0.38(0.59-2.0 \AA)$ with two selenium sites. Density modification with RESOLVE (Terwilliger, 2003) was followed by automated model building using ARP/wARP (Cohen et al., 2004). Model completion and refinement were performed with Coot (Emsley \& Cowtan, 2004) and REFMAC 5.5 (Winn et al., 2003) using the remote $\left(\lambda_{1}\right)$ data. The refinement included phase restraints from SOLVE and TLS refinement with four TLS groups per chain. Datacollection and refinement statistics are summarized in Table 1.

\subsection{Identification of metal-binding sites}

$\mathrm{X}$-ray fluorescence emission peaks for selenium, arsenic, zinc and nickel were observed when the crystal was excited with X-rays $500 \mathrm{eV}$ above the Se edge on SSRL beamline 11-1. In order to determine the identity of the metals at the individual sites in the structure, four additional data sets were collected on SSRL beamline 1-5. These data sets were collected to $2.9 \AA$ resolution at wavelengths of $1.278,1.292$, 1.480 and $1.497 \AA$, which are above and below the zinc and nickel absorption edges. Data statistics are described in Table 2. Anomalous difference Fourier maps were calculated for each wavelength using the density-modified experimental MAD phases. The large changes in peak heights across the zinc or nickel absorption edge clearly showed that one site contained zinc and the other contained nickel. The integrated peak heights at the metal sites are shown with the peak heights for the selenium and sulfur sites as a reference in Table 3. 
Table 2

Data-collection statistics for metal-site identification.

Values in parentheses are for the highest resolution shell.

\begin{tabular}{lllll}
\hline & $\lambda_{4}$ above $\mathrm{Zn}$ & $\lambda_{5}$ below $\mathrm{Zn}$ & $\lambda_{6}$ above Ni & $\lambda_{7}$ below Ni \\
\hline Wavelength $(\AA)$ & 1.2782 & 1.2915 & 1.4795 & 1.4974 \\
Resolution range $(\AA)$ & $45.1-2.90$ & $45.1-2.90$ & $45.1-2.90$ & $45.1-2.90$ \\
& $(2.98-2.90)$ & $(2.98-2.90)$ & \multicolumn{1}{c}{$(2.98-2.90)$} & \multicolumn{1}{c}{$(2.98-2.90)$} \\
No. of observations & 17029 & 16952 & 15853 & 15655 \\
No. of unique reflections & 4658 & 4658 & 4527 & 4489 \\
Completeness (\%) & $99.7(99.6)$ & $99.8(98.9)$ & $97.0(77.2)$ & $96.1(72.5)$ \\
Mean $I / \sigma(I)$ & $47.4(32.8)$ & $51.8(35.7)$ & $50.2(28.9)$ & $50.8(27.1)$ \\
$R_{\text {merge }}$ on $I^{\dagger}(\%)$ & $2.9(4.0)$ & $2.2(3.3)$ & $2.3(3.1)$ & $2.2(3.0)$ \\
\hline
\end{tabular}

$\dagger R_{\text {merge }}=\sum_{h k l} \sum_{i}\left|I_{i}(h k l)-\langle I(h k l)\rangle\right| / \sum_{h k l} \sum_{i} I_{i}(h k l)$.

The theoretical $f^{\prime \prime}$ values at each wavelength are listed for comparison in Table 4.

\subsection{Validation and deposition}

The quality of the crystal structure was analyzed using the JCSG Quality Control server (http://smb.slac.stanford.edu/jcsg/QC). This server processes the coordinates and data using a variety of validation tools including AutoDepInputTool (Yang et al., 2004), MolProbity (Davis et al., 2007), WHATIF 5.0 (Vriend, 1990), RESOLVE (Terwilliger, 2003) and MOLEMAN2 (Kleywegt, 2000) as well as several in-house scripts and summarizes the output. Fig. 1(b) was adapted from an analysis using PDBsum (Laskowski et al., 2005) and all other figures were prepared with PyMOL (DeLano Scientific). Atomic coordinates and experimental structure factors for Jann_2411 have been deposited in the PDB (http://www.pdb.org) under accession code $3 \mathrm{~h} 0 \mathrm{n}$.

\section{Results and discussion}

\subsection{Overall structure}

The crystal structure of Jann_2411 (Fig. 1a) was determined to $1.45 \AA$ resolution using the MAD method. Data collection and refinement statistics are summarized in Table 1. The final model included one protomer (residues 1-184), three acetate molecules, two glycerol molecules, one zinc ion, one nickel ion, two sodium ions and 240 water molecules in the asymmetric unit. Arg185, Ala186 and Thr187 at the C-terminus and Gly0 remaining after the cleavage of the expression/purification tag at the $\mathrm{N}$-terminus were disordered and not modeled. Poor electron density was observed for the side chains of Lys45, Arg161, Asn162, Lys177 and Arg182. The side chains of the following residues were modeled in two conformations: Ile37, Asp42, Arg67, Asp74, His90, Gln103, Leu116, Glu119, Leu120 and Met123. The Matthews coefficient ( $V_{\mathrm{M}}$; Matthews, 1968 ) was $2.5 \AA^{3} \mathrm{Da}^{-1}$ and the estimated solvent content was $50.9 \%$. The Ramachandran plot produced by MolProbity (Davis et al., 2007) showed that $98.9 \%$ of the residues were in favored regions, with no outliers.

Jann_2411 belongs to the Pfam family known as DUF1470, which accounts for the entire length of the protein sequence. However, the structure shows that Jann_2411 is actually comprised of two domains (Figs. $1 a$ and $2 a$ ). The first domain (residues 1-142) can be visualized as two subdomains $(\mathrm{H} 2-\mathrm{H} 4, \beta 1-\beta 2$ and $\mathrm{H} 5-\mathrm{H} 7, \beta 3-\beta 4)$ that share similar topology and secondary-structure elements, namely a helix$\beta$-hairpin-helix motif (H2- $\beta 1-\beta 2-\mathrm{H} 3$ in the first subdomain; H6- $\beta 3$ $\beta 4-\mathrm{H} 7$ in the second subdomain), with an additional helix (H4 from the first subdomain and $\mathrm{H} 5$ from the second subdomain) linking the two motifs. We have therefore named this region the ABATE domain, representing the Alpha-Beta-hairpin-Alpha TandEm motif.
Table 3

Anomalous difference Fourier integrated peak heights.

The maps were calculated with data from 20 to $2.9 \AA$. The signal listed is the value reported by MAPMAN (Kleywegt \& Jones, 1996) after integration of a sphere of radius $2 \AA$ around the atom center from the final refined model. Sulfur and selenium sites are listed to provide a reference for differences in scale between different maps.

\begin{tabular}{lcclll}
\hline Atom & $\lambda_{1}$ MADSe & $\lambda_{4}$ above $\mathrm{Zn}$ & $\lambda_{5}$ below $\mathrm{Zn}$ & $\lambda_{6}$ above $\mathrm{Ni}$ & $\lambda_{7}$ below Ni \\
\hline Se-1 & 11.26 & 2.96 & 1.57 & 2.93 & 3.13 \\
Se-123a & $21.11 \dagger$ & 5.64 & 3.50 & 4.43 & 4.23 \\
Se-123b & $21.84 \dagger$ & 5.91 & 3.60 & 4.47 & 4.30 \\
Zn & 15.69 & 17.43 & 2.92 & 3.97 & 4.11 \\
Ni-a & $5.07 \dagger$ & 5.49 & 5.27 & 4.59 & 0.42 \\
Ni-b & $3.88 \dagger$ & 3.07 & 2.84 & 3.34 & 0.39 \\
S-35 & 0.25 & 0.70 & 1.02 & 1.58 & 1.84 \\
S-128 & 0.48 & 1.10 & 1.30 & 2.12 & 2.30 \\
S-147 & 3.44 & 2.51 & 1.86 & 2.21 & 2.72 \\
S-152 & 2.44 & 2.88 & 1.48 & 2.35 & 2.03 \\
S-168 & 3.23 & 3.55 & 1.45 & 2.38 & 2.79 \\
S-172 & 5.47 & 5.11 & 2.29 & 2.59 & 2.77 \\
\hline
\end{tabular}

$\dagger$ The Se atom from residue A123 and the $\mathrm{Ni}$ atom from residue A202 were modeled in alternate conformations and each partial occupancy site was integrated separately without overlap removal.

Table 4

Theoretical $f^{\prime \prime}$ values at each wavelength.

Theoretical $f^{\prime \prime}$ data were extracted from tables compiled by Ethan Merritt (http:// skuld.bmsc.washington.edu/scatter/AS_periodic.html).

\begin{tabular}{llllll}
\hline Data set & $\lambda_{1}$ MADSe & $\lambda_{4}$ above $\mathrm{Zn}$ & $\lambda_{5}$ below $\mathrm{Zn}$ & $\lambda_{6}$ above Ni & $\lambda_{7}$ below Ni \\
\hline Wavelength $(\AA)$ & 0.9184 & 1.2782 & 1.2915 & 1.4795 & 1.4974 \\
Energy $(\mathrm{eV})$ & 13500 & 9700 & 9600 & 8380 & 8280 \\
Theoretical $f^{\prime \prime} \mathrm{Se}$ & 3.37 & 0.81 & 0.83 & 1.06 & 1.08 \\
Theoretical $f^{\prime \prime} \mathrm{Zn}$ & 2.23 & 3.86 & 0.49 & 0.63 & 0.64 \\
Theoretical $f^{\prime \prime} \mathrm{Ni}$ & 1.75 & 3.05 & 3.10 & 3.87 & 0.48 \\
Theoretical $f^{\prime \prime} \mathrm{S}$ & 0.21 & 0.39 & 0.40 & 0.52 & 0.53 \\
\hline
\end{tabular}

The helices of the first subdomain ( $\mathrm{H} 2$ and $\mathrm{H} 3)$ are stacked perpendicular to helices $\mathrm{H} 5$ and $\mathrm{H} 7$ which, together with $\mathrm{H} 6$, form a helical bundle capped on one end by the $3_{10}$-helix $\mathrm{H} 8$ and on the other by helix H4. In both subdomains, the $\beta$-hairpin is oriented orthogonally with respect to the long axes of the helices in the ABATE motif. Superposition of the two ABATE motifs (residues 946 for ABATE1 and 80-139 for ABATE2) results in an r.m.s.d. of $3.2 \AA$ over 27 residues ( $3 \%$ identity), which is non-significant. Additionally, sequence alignment shows little residue conservation in this domain, with no strictly conserved residues observed between representative ABATE-family sequences (Fig. 3), suggesting that this domain evolved as a single unit as opposed to the gene-duplication event that might be suggested by the presence of two ABATE motifs.

The second domain (residues 143-187; H8, $\beta 5-\beta 6$, H9) forms a treble-clef zinc finger (Fig. 2). The zinc ion is coordinated by two cysteines (Cys147 and Cys152) from a loop termed the zinc knuckle, located between the strands of the third $\beta$-hairpin $(\beta 5-\beta 6)$, and two cysteines from the N-terminus of helix H9 (Cys168 and Cys172) (Fig. 2a). This arrangement of zinc-coordinating residues is typical of treble-clef zinc fingers (Grishin, 2001; Krishna et al., 2003). Other strictly conserved residues (Fig. 3) in this domain include Asp158 and Arg175. A high degree of conservation is observed for a number of positively charged residues (Arg143, Arg161, Arg165, Lys177, Arg182 and Arg184), suggesting that this region could present a nucleic acid binding site. Furthermore, residues 146 (a hydrophobic residue) and 167 (an aromatic residue) are highly conserved (Fig. 3) and could intercalate between the DNA bases. Based on the most conserved motif found in the C-terminal $\alpha$-helix in this family of proteins, we have named this domain the CGNR zinc finger. The actual amino-acid sequence in Jann_2411 is CQNR. 
The above findings led to the re-evaluation of the Pfam DUF1470 family which, as a result of our study, will now be split into two entries in the next Pfam release (the current release is Pfam 23.0, July 2008). The original DUF1470 entry has been truncated and renamed to represent the ABATE domain, while a new Pfam family has been created for the C-terminal CGNR zinc-finger domain called zf-CGNR (Pfam accession PF11706).

\subsection{Similarity to other proteins}

SCOP classifies Jann_2411 as an $\alpha+\beta$ protein with an unusual fold (http://scop.mrc-lmb.cam.ac.uk/scop/data/scop.b.e.dda.b.b.b.html). A search with FATCAT (Ye \& Godzik, 2004) using the N-terminal domain of Jann_2411 gave some hits involving helices H5-H7, but these structures [a three-helix bundle from a bacterial ATPase (PDB code $2 \mathrm{v} 6 \mathrm{y}$ ) and a histidine phosphotransferase domain (PDB code $1 \mathrm{sr} 2)$ ] involved less than half of the domain and displayed diverse functionalities. No single hit was found for the N-terminal domain in its entirety, leading us to propose that this domain represents a new fold. The C-terminal domain was structurally similar (main-chain r.m.s.d. of $2.5 \AA$ over 40 residues with a sequence identity of $11 \%$ ) to a plant homeodomain (PHD) finger from yeast (PDB code 2jmi), confirming the identity of this domain as a treble-clef zinc finger. Superposition of $\mathrm{H} 9$ onto the corresponding helix of the yeast structure revealed that the arrangement of the zinc ion and coordinating cysteines is conserved between the two structures (Fig. 2b).

Analysis of the crystallographic packing of Jann_2411 using the PISA server (Krissinel \& Henrick, 2007) and analytical size-exclusion chromatography in combination with static light scattering indicate that a dimer is the likely quaternary form. The crystallographic dimer interface mainly involves hydrophobic contacts from the second $\beta$-hairpin (strands $\beta 3-\beta 4$ ), helices $\mathrm{H} 6$ and $\mathrm{H} 7$ and the intervening loops, and buries $990 \AA^{2}$ of surface area per monomer (Fig. $2 a$ ). This arrangement results in the formation of a deep cavity $\left(\sim 2800 \AA^{3}\right.$
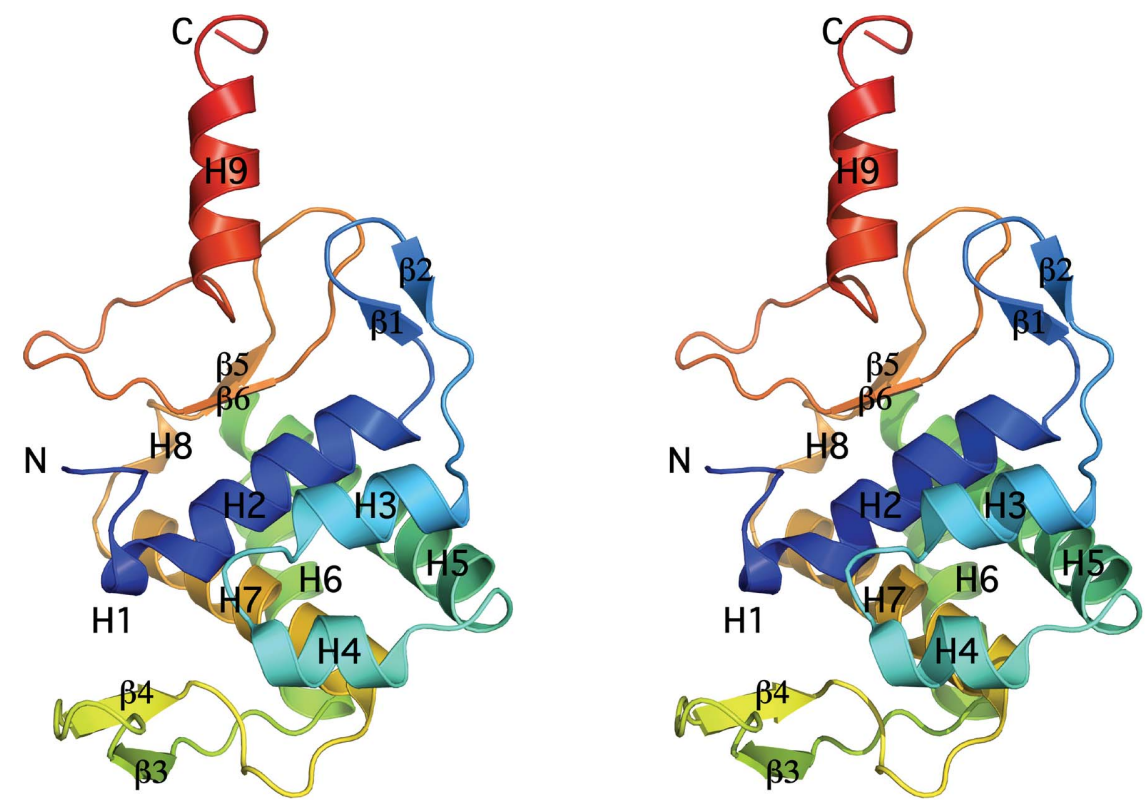

(a)

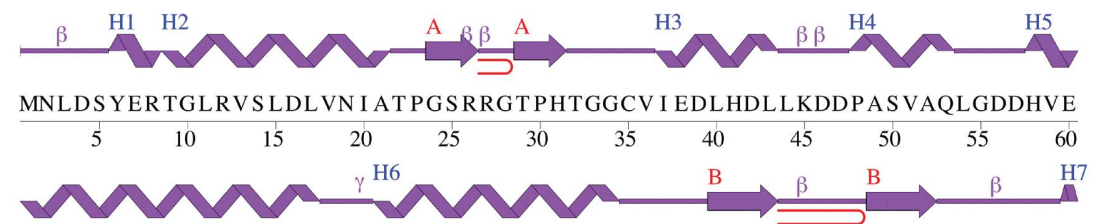

GFVELARL LHT A I DAL S NGQVAT AAT ALNHL LRKHPAT P ELAQDPDGTWRLHHHPLDAEL
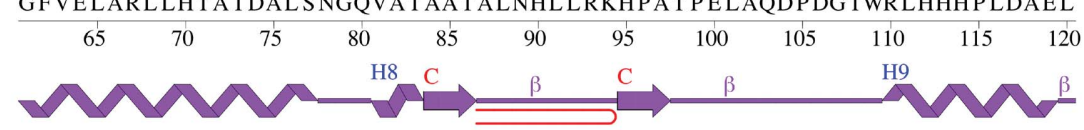

V PMWT A I CAEGLARE I GHQNVRR FG I CNAHR CDR VYFDT S RNGTRQYC S LACQNR VKAAA

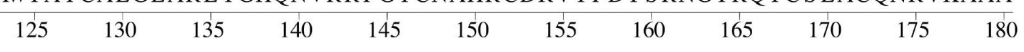

$\beta \beta$

FRER

Figure 1

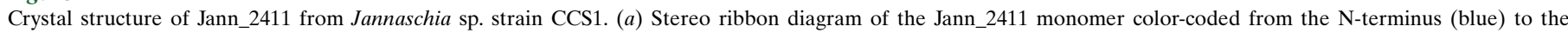

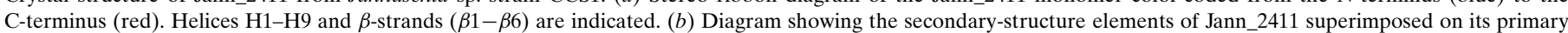

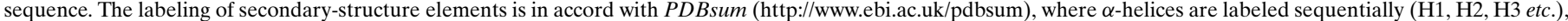

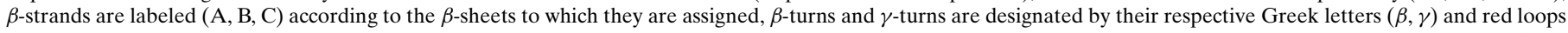

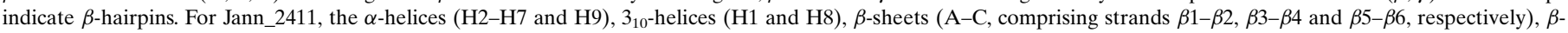
turns $(\beta)$ and $\beta$-hairpins are indicated. 
according to the CastP server; Binkowski et al., 2003) along the dimer interface, delimited by the long loop connecting the last hairpin (strands $\beta 5-\beta 6$ ) and helix H9. However, sequence and structure conservation in this domain is very weak, making quaternary states difficult to infer for the rest of the family.

Treble-clef zinc fingers are usually incorporated into larger structures and are found in proteins with a wide range of functions, many of which involve transcriptional regulation (Grishin, 2001; Krishna et al., 2003). Jannaschia sp. CCS1 is a member of the Roseobacter lineage, a taxon of marine bacteria. CCS1 is a phototroph that uses bacteriochlorophyll to harvest energy from light without the formation of oxygen. Genes predicted to have functional associations with Jann_2411 in the STRING database (http://string.embl.de) include a transmembrane protein of unknown function (Jann_2410) and the transcriptional regulator Jann_2412, a member of the Asr gene family. The Asr gene family is widespread in higher plants and most members of this family are up-regulated under a range of environmental stress conditions; their products are thought to function as transcriptional regulators (Frankel et al., 2006).

Other members of this newly defined ABATE protein family are found in plant symbionts (Rhizobium, Bradyrhizobium) and plant pathogens (Streptomyces, Ralstonia, Agrobacterium); they are around 180 residues in length and also contain the newly designated zf-CGNR (Pfam accession PF11706). Genome-location analysis of representative ABATE sequences shows co-occurrence with putative DNA-binding proteins, transcriptional regulators and membrane proteins implicated in ABC transport. In Streptomyces, several of the proteins co-occurring with ABATE homologs, such as sporulationspecific cell-division proteins and RNA polymerase sigma factors, are implicated in the control of aerial mycelium development (Dalton $e t$ al., 2007; Gordon et al., 2008) and are activated under conditions of cell-envelope stress, such as hyperosmolarity (Kormanec \& Sevcikova, 2002).

A number of molecular mechanisms are shared between symbionts and pathogens, especially those involving host colonization and adaptation to a particular ecological niche (Hentschel et al., 2000). In both instances, changes in ecological and host environments necessitate fast adaptation strategies on the part of the microorganism. Zinc fingers possess the functional versatility necessary for this adaptation and have been exploited may times by nature and by the pharmaceutical industry (Papworth et al., 2006). The molecular function of the ABATE domain remains elusive. However, given the
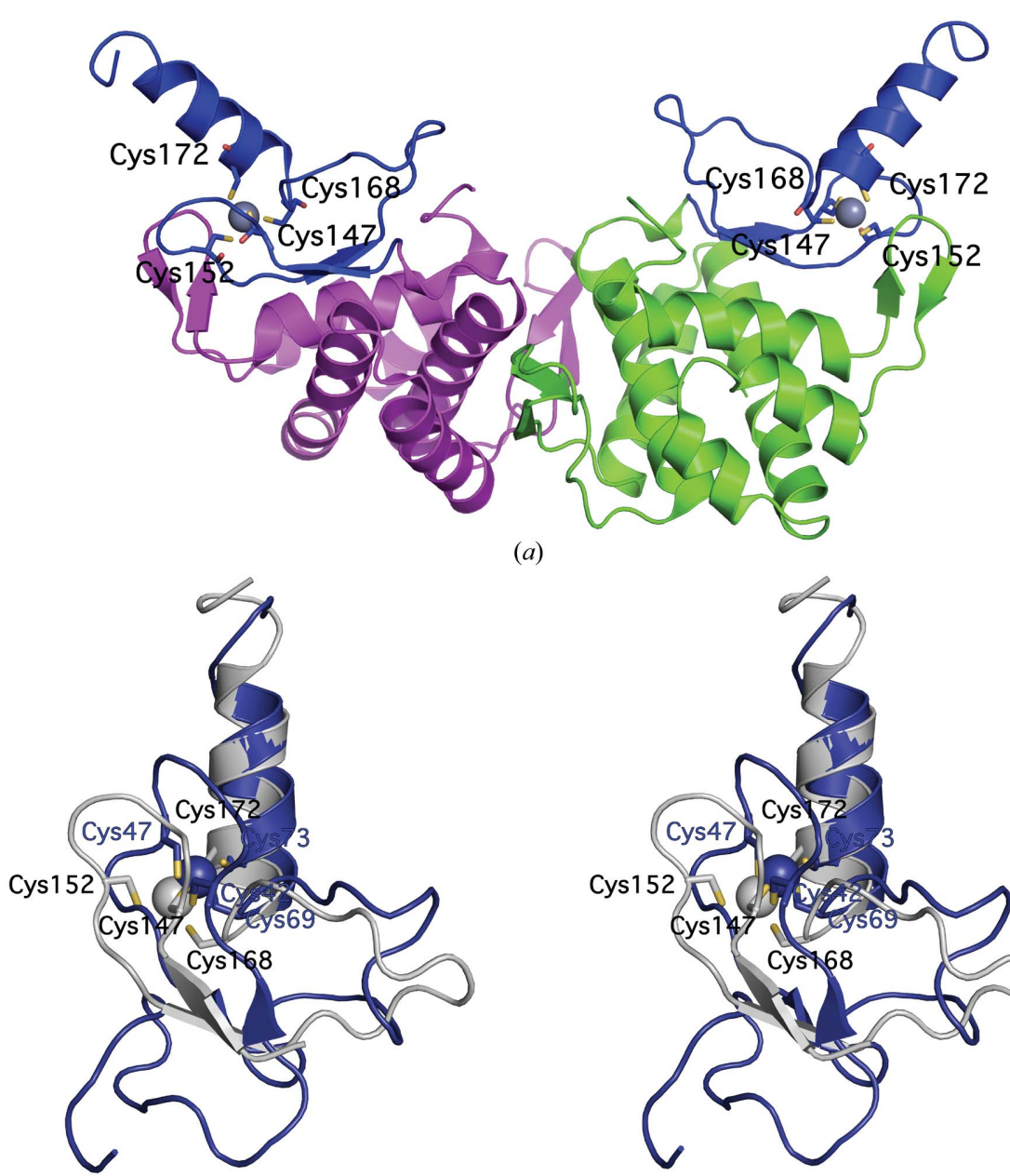

(a)

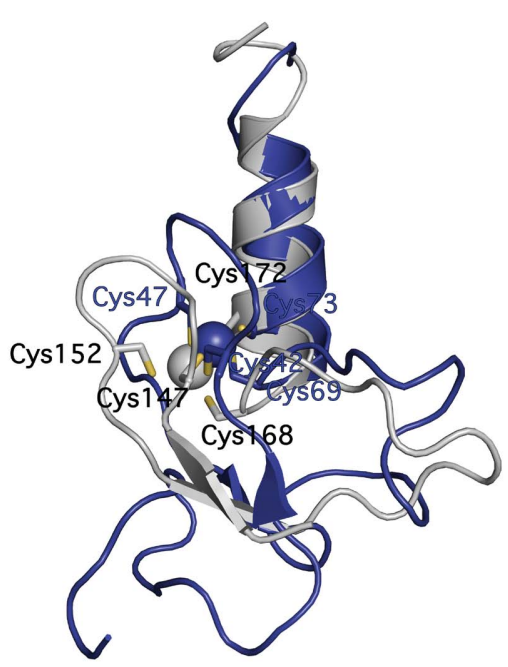

(b)

Figure 2

The C-terminal domain of Jann_2411 forms a zinc finger. (a) Ribbon representation of the Jann_2411 dimer. The zinc-finger domains are depicted in blue, the N-terminal domains are depicted in magenta and green and the zinc ions are shown as gray spheres. The zinc-coordinating cysteines are shown in ball-and-stick representation and labeled. (b) Stereoview of the structural superposition of the C-terminal domain of Jann_2411 (PDB code 3h0n, residues 144-187, gray) and a PHD finger fragment from yeast Yng1 protein (PDB code 2jmi, residues 38-83, blue). Zinc ions are shown as spheres and side chains of coordinating residues are indicated. 


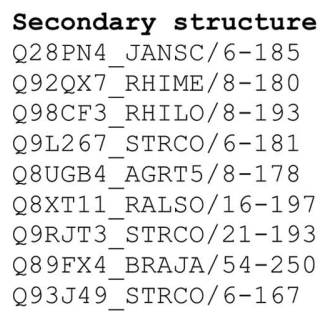

Secondary structure Q28PN4 JANSC/6-185 Q920X7_RHIME/8-180 Q98CF3-RHILO/8-193 Q9L267 STRCO/6-181 Q8UGB4-AGRT5/8-178 Q8XT11 RALSO/16-197 Q9RJT3_STRCO/21-193 Q89FX4 BRAJA/54-250 Q93J49_STRCO/6-167

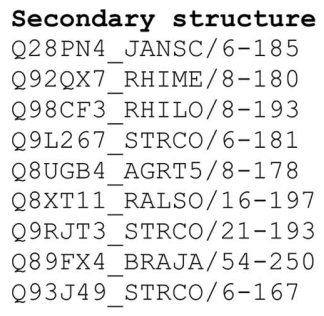

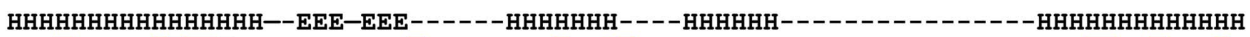
YERTGLRVSLDLVNIATPGSRRGTPHTGGCVIEDLHDLLKDDPASVAQ-----------LGDDHVEGFVELARLLH HRFSGGALALDVANSVVMRFDAERRIDRFDDPAAIDTFAEAANLYGAE---RNRFGTLVPAKSERRNAFIGLREATD HRFTGGLLALDTANTVVLRGDPDRTFDRFDDPAEIARFADAASGFRAA--ELGDRRLTVSSPTAIAPVVLSIRETTD DTRCALDTVVDLVNTVP---EDESAPDGLPDVAALQEFVGTHEISDVG-----------VLTEFDLSAVRRIRARFA HRFAGGALALDVANSVILRSDAARTLDRFSVPEQIVTFAEAATRLGME---RDRFPALVAPEADKRPIFLELREAID PAS IADH PVLDMLNTVAN--VNGQPHDFWQADEDVSAWLMQAGWVDET-------LAGRYPPGALLAVARHLREVIR FAFVSGNPALDLTGTVLS--RRDEPVDLLAVPADLGRWVAACEGLPDR----------VTATPSAFDAALNLREAVY AMF IADSLGLDFLNSVAT--PVDTPVDWIDDGDGL I DWLEQAKLVPADELHALKARAAPGELDEVADQARALREWFR PAPGGLGLVETLVNTL----DLETGADSLDTEEGRAP--------------------PGLTEDDVPAARELRESLR

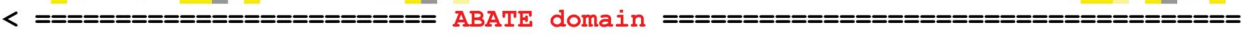

HHHHHHH-------HHHHHHHHHHHHHH-----EEEE-------EEEE-------HHHHHHHHHHHHHHHHHH-TAIDALSNGQ-----VATAATALNHLLRKHPATPELAQDPDGT--WRLHHHPLDAELVPMWTAICAEGLAREIGHQN RHFRALV-------RTEDRPDLLADLLEA IATVLRQPADTRK-------------GTPLDVATAHSALGLIAGPE RLFRGAVSKGAV---ATADLPDFLRACAEGLARSRTEVGAPGR-------PFGDPLVPI PFEAALAVSALSLLRDDT AVFAAPD-------ARTAAGLINDLVAAAGTTPRLTDHDGYD--WH I HYFAPGASVADHLTADCGMALAF FVVAGE DHFRSAV-------SRGADDDGKLARLLSVGGTALRAFPTSE-------------SLANATVHSALSLLAGET TLVQTRKAG------KRANPDALNVFLRQAPSHPALVWQGDAP---PRLERRRPADSVEQCLAPLAESAAQLIADGD RLALDRVLDRRF---HIPSLEVVNAAAAGPLPTVRLGDAGVRM-----------SGGLPAVLTHVARSGIAVLADPG GFVLEHA-GRPLTADALRELGPLNGLLEREESFRQIEPGHGDVGGVLKLQTKRRWRSAESLLLPVGEALATFVCDED ATLLAHA-GHPP----HRAVTPLGELLAAAPLVVAVDPADGSA--------ALAPAGEGSLTARVAAAVAQALVAGT

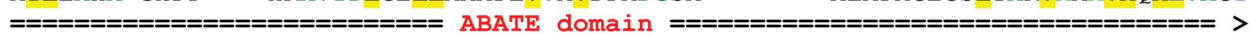

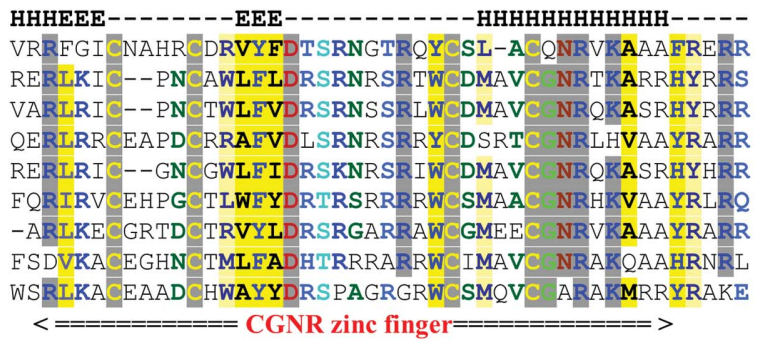

Figure 3

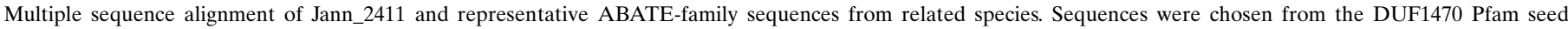

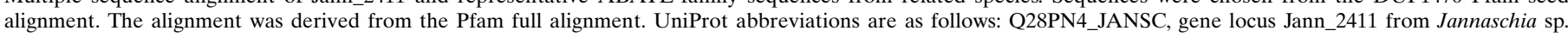

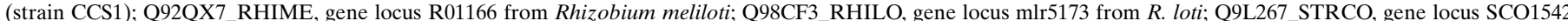

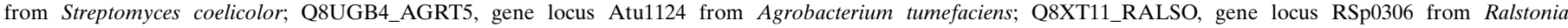

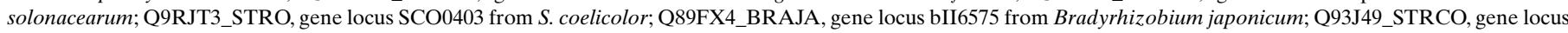
SCO3054 from S. coelicolor. Residues are colored by conservation using the CHROMA software with default settings (Goodstadt \& Ponting, 2001).

prediction that the C-terminal domain binds DNA, then the $\mathrm{N}$-terminal domain may allow the protein to act as a signal-dependent transcriptional regulator with the ABATE domain conferring sensitivity to some as yet undefined ligand. This combination of a DNAbinding domain with a ligand-sensing domain is a prevalent form of regulation of operons in bacteria, such as the lactose or arabinose operons (Anantharaman et al., 2001). The likely dimeric nature of the protein and the long loop delimiting the suggested DNA-binding region hint at the possibility of an allosteric mechanism that might abolish DNA binding upon binding to an as yet unknown ligand. The LacI protein represents a canonical example for such an allosteric transition, with ligand-binding inducing a hinge-like motion that alters the relative subdomain orientations within the dimer, thereby changing DNA affinity (Lewis et al., 1996). Many other similar examples exist, such as TetR (Henssler et al., 2005; Premkumar et al., 2007; Koclega et al., 2007), all of which form dimers or higher order oligomers.

The availability of additional ABATE sequences and structures should shed light on the evolutionary history of this protein family. The information presented here, in combination with further biochemical and biophysical studies, should yield valuable insights into the functional role of Jann_2411. Models of Jann_2411 homologs can be accessed at http://www1.jcsg.org/cgi-bin/models/get_mor.pl? key $=3 \mathrm{~h} 0 \mathrm{nA}$.
Additional information about the protein described in this study is available from TOPSAN (Krishna et al., 2010) http://www.topsan.org/ explore?PDBid=3h0n.

\section{Conclusions}

The first structural representative of the DUF1470 family revealed a two-domain organization, with the $\mathrm{N}$-terminal domain presenting a new fold and the C-terminal domain consisting of a treble-clef zinc finger. The structure additionally allowed a re-evaluation of the Pfam signature and the Pfam assignment and suggests a role for this family in stress-induced transcriptional regulation.

This work was supported by the National Institutes of Health, Protein Structure Initiative grant Nos. P50 GM62411 and U54 GM074898. Portions of this research were carried out at the Stanford Synchrotron Radiation Lightsource (SSRL). The SSRL is a national user facility operated by Stanford University on behalf of the US Department of Energy, Office of Basic Energy Sciences. The SSRL Structural Molecular Biology Program is supported by the Department of Energy, Office of Biological and Environmental Research and by the National Institutes of Health (National Center for Research Resources, Biomedical Technology Program and the 
National Institute of General Medical Sciences). The content is solely the responsibility of the authors and does not necessarily represent the official views of the National Institute of General Medical Sciences or the National Institutes of Health. Genomic DNA from Jannaschia sp. CCS1 was a gift from Professor Alison Buchan at the University of Tennessee, Knoxville. AB is supported by the Wellcome Trust (grant No. WT077044/Z/05/Z).

\section{References}

Anantharaman, V., Koonin, E. V. \& Aravind, L. (2001). J. Mol. Biol. 307, 1271-1292.

Binkowski, T. A., Naghibzadeh, S. \& Liang, J. (2003). Nucleic Acids Res. 31, 3352-3355.

Cohen, A. E., Ellis, P. J., Miller, M. D., Deacon, A. M. \& Phizackerley, R. P. (2002). J. Appl. Cryst. 35, 720-726.

Cohen, S. X., Morris, R. J., Fernandez, F. J., Ben Jelloul, M., Kakaris, M., Parthasarathy, V., Lamzin, V. S., Kleywegt, G. J. \& Perrakis, A. (2004). Acta Cryst. D60, 2222-2229.

Collaborative Computational Project, Number 4 (1994). Acta Cryst. D50, 760-763.

Dalton, K. A., Thibessard, A., Hunter, J. I. \& Kelemen, G. H. (2007). Mol. Microbiol. 64, 719-737.

Davis, I. W., Leaver-Fay, A., Chen, V. B., Block, J. N., Kapral, G. J., Wang, X., Murray, L. W., Arendall, W. B. III, Snoeyink, J., Richardson, J. S. \& Richardson, D. C. (2007). Nucleic Acids Res. 35, W375-W383.

Emsley, P. \& Cowtan, K. (2004). Acta Cryst. D60, 2126-2132.

Finn, R. D., Tate, J., Mistry, J., Coggill, P. C., Sammut, S. J., Hotz, H. R., Ceric, G., Forslund, K., Eddy, S. R., Sonnhammer, E. L. \& Bateman, A. (2008). Nucleic Acids Res. 36, D281-D288.

Frankel, N., Carrari, F., Hasson, E. \& Iusem, N. D. (2006). Gene, 378, 74-83. Goodstadt, L. \& Ponting, C. P. (2001). Bioinformatics, 17, 845-846.

Gordon, N. D., Ottaviano, G. L., Connell, S. E., Tobkin, G. V., Son, C. H., Shterental, S. \& Gehring, A. M. (2008). J. Bacteriol. 190, 894-904.

Grishin, N. V. (2001). Nucleic Acids Res. 29, 1703-1714.

Henssler, E. M., Bertram, R., Wisshak, S. \& Hillen, W. (2005). FEBS J. 272, $4487-4496$.
Hentschel, U., Steinert, M. \& Hacker, J. (2000). Trends Microbiol. 8, 226-231.

Kabsch, W. (1993). J. Appl. Cryst. 26, 795-800.

Kleywegt, G. J. (2000). Acta Cryst. D56, 249-265.

Kleywegt, G. J. \& Jones, T. A. (1996). Acta Cryst. D52, 826-828.

Koclega, K. D., Chruszcz, M., Zimmerman, M. D., Cymborowski, M., Evdokimova, E. \& Minor, W. (2007). J. Struct. Biol. 159, 424-432.

Kormanec, J. \& Sevcikova, B. (2002). Mol. Genet. Genomics, 267, 536-543.

Krishna, S. S., Majumdar, I. \& Grishin, N. V. (2003). Nucleic Acids Res. 31, 532-550.

Krishna, S. S., Weekes, D., Bakolitsa, C., Elsliger, M.-A., Wilson, I. A., Godzik, A. \& Wooley, J. (2010). Acta Cryst. F66, 1143-1147.

Krissinel, E. \& Henrick, K. (2007). J. Mol. Biol. 372, 774-797.

Laskowski, R. A., Chistyakov, V. V. \& Thornton, J. M. (2005). Nucleic Acids Res. 33, D266-D268.

Lesley, S. A. et al. (2002). Proc. Natl Acad. Sci. USA, 99, 11664-11669.

Lewis, M., Chang, G., Horton, N. C., Kercher, M. A., Pace, H. C., Schumacher, M. A., Brennan, R. G. \& Lu, P. (1996). Science, 271, 1247-1254.

Matthews, B. W. (1968). J. Mol. Biol. 33, 491-497.

McPhillips, T. M., McPhillips, S. E., Chiu, H.-J., Cohen, A. E., Deacon, A. M., Ellis, P. J., Garman, E., Gonzalez, A., Sauter, N. K., Phizackerley, R. P., Soltis, S. M. \& Kuhn, P. (2002). J. Synchrotron Rad. 9, 401-406.

Papworth, M., Kolasinska, P. \& Minczuk, M. (2006). Gene, 366, 27-38.

Premkumar, L. et al. (2007). Proteins, 68, 418-424.

Santarsiero, B. D., Yegian, D. T., Lee, C. C., Spraggon, G., Gu, J., Scheibe, D., Uber, D. C., Cornell, E. W., Nordmeyer, R. A., Kolbe, W. F., Jin, J., Jones, A. L., Jaklevic, J. M., Schultz, P. G. \& Stevens, R. C. (2002). J. Appl. Cryst. 35, $278-281$.

Sheldrick, G. M. (2008). Acta Cryst. A64, 112-122.

Terwilliger, T. C. (2003). Acta Cryst. D59, 1174-1182.

Terwilliger, T. C. \& Berendzen, J. (1999). Acta Cryst. D55, 849-861.

Tickle, I. J., Laskowski, R. A. \& Moss, D. S. (1998). Acta Cryst. D54, $243-$ 252.

Vriend, G. (1990). J. Mol. Graph. 8, 52-56.

Winn, M. D., Murshudov, G. N. \& Papiz, M. Z. (2003). Methods Enzymol. 374, 300-321.

Yang, H., Guranovic, V., Dutta, S., Feng, Z., Berman, H. M. \& Westbrook, J. D. (2004). Acta Cryst. D60, 1833-1839.

Ye, Y. \& Godzik, A. (2004). Nucleic Acids Res. 32, W582-W585. 